\title{
Internet Network in Space for Small Satellites: Concept and Experiments
}

\author{
Pirada Techavijit, ${ }^{1 *}$ Polkit Sukchalerm, ${ }^{2}$ Natthapong Wongphuangfuthaworn, ${ }^{2}$ \\ Aniwat Plodpai, ${ }^{2}$ Shariff Manuthasna, ${ }^{2}$ and Sorawat Chivapreecha ${ }^{1}$ \\ ${ }^{1}$ Department of Telecommunication Engieneering, Faculty of Engineering, \\ King Mongkut's Institute of Technology Ladkrabang, Bangkok 10520, Thailand \\ ${ }^{2}$ Thailand Space and Aeronautics Research (TSR), \\ 81/741 Prachautid79 Thung Kru, Bangkok 10140, Thailand
}

(Received December 18, 2017; accepted July 23, 2018)

Keywords: Internet in space, nanosatellite, high-altitude platform, CubeSat, LoRaWAN, satellite communication

Currently, no wireless network service is being offered in space. This fact has caused satellite developers to establish their own wireless network with a low coverage area that does not function very well. These problems can be resolved by setting up an effective wireless network that can be offered as a service to satellites in orbit. This study is focused on designing a high-quality wireless-in-space network to connect satellites in orbit to the Internet. Such a network would enable satellites to be controlled through the Internet from the Earth without having their own ground stations. In this paper, various aspects of the design of a network setup standard are discussed. These aspects include appropriate wireless communication and the construction of a model satellite that can be connected to the network from space. The work also included testing the design by setting up the Internet network for use above Thailand's territory and launching the original model satellite for the demonstration of the principle. The sensor data was then transmitted in real time to audiences from all over the country and used to design an Internet network in space with global coverage, which is a target for further development.

\section{Introduction}

Over the past five years, the number of low earth orbit (LEO) satellites has increased significantly. This is especially true for nanosatellites ${ }^{(1,2)}$ that weigh only $1-50 \mathrm{~kg}$ and are much smaller than previous satellites. This is possible because of advancements in computer and electronics technology in recent years. As a result, the number of nanosatellites launched to orbit has tripled from 88 in 2016 to 292 in 2017. ${ }^{(3)}$

However, there are still some challenges in communication systems for nanosatellites because no wireless network service is offered in space. Therefore, satellite developers often have to create their own satellite communication system from scratch, including RF modulation *Corresponding author: e-mail: ptechavi@gmail.com https://doi.org/10.18494/SAM.2018.1854 
and protocol settings. Unfortunately, their development has not shown promising results. It has been reported that $81 \%$ of the systems still have data transmission modes with data rates lower than $10 \mathrm{kbps}$. These systems are also the main cause of failure in $49 \%$ of the nanosatellites. ${ }^{(4)}$ Moreover, only specific pairs of ground stations and satellites are compatible and can successfully communicate with each other. As a result, the coverage area of the communication is very limited and a ground station can communication with its satellite counterpart for only 40 min per day.

Such problems will be resolved if a highly efficient central network that offers service in space is available. The existing network with the highest potential is the Internet. $^{(5,6)}$ Its capacity is so advanced that all types of protocols are available for transmitting different kinds of data, including hyper text transfer protocol (HTTP), file transfer protocol (FTP), and realtime streaming protocol (RTSP). Additionally, the physical layer aspect of the Internet has been so highly developed that it can connect the entire world. More importantly, commercial off-theshelf (COTS) systems have been designed to be very user-friendly and widely adopted.

This study aims at designing and developing the concept of expanding the Internet into space. A satellite could then directly connect to the Internet using a global standard protocol while orbiting in space. This would enhance communication and enable ubiquitous communication between ground stations and satellites in orbit. Furthermore, the study has resulted in the design of a model satellite to test this concept and launched it into near-space to demonstrate the operation of the entire system. The results will allow us to improve our idea and develop an Internet with global coverage in the future.

\section{Satellite Communication: Traditional and New Concept Design}

Most nanosatellites orbit in LEO at an altitude of $2000 \mathrm{~km}$ or less. Their orbital periods usually take 84-127 min. As the satellites keep changing their locations while orbiting, they enter the visibility region of their ground receiving station 3-4 times per day. During this time, the communication between the stations and the satellites is enabled through a wireless connection by using radio waves.

\subsection{Traditional method of satellite communication}

In the past, no wireless network services were offered in space because the number of orbiting satellites was small. Therefore, satellite developers needed to develop their own communication system under some constraints. Thus, the satellite developers have not achieved good results. Some satellites may provide service to other receivers. However, usually only one control station exists and its communication characteristic can be described as follows (Fig. 1).

\subsubsection{Various modes of modulation and data transmission speed}

According to a statistical survey on the satellite CubeSat, which accounts for $90 \%$ of the nanosatellites, a great variety of modes of communication were reported as shown in Fig. 2. 


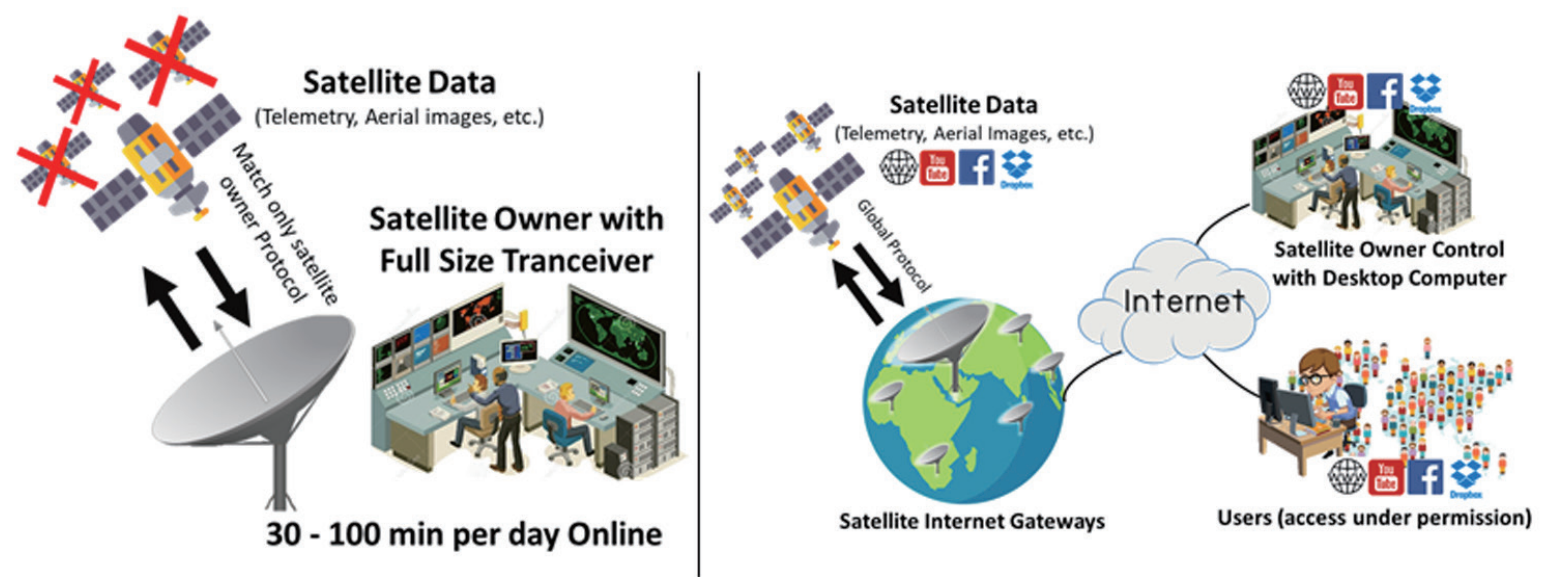

Fig. 1. (Color online) Traditional vs new concept of communication.

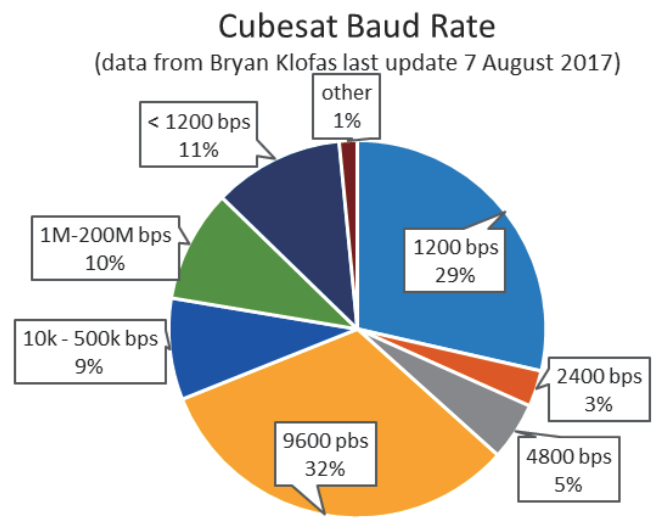

(a)



(b)

Fig. 2. (Color online) (a) Statistics for CubeSat Baud rate and (b) statistics for CubeSat modulation. (3,7) $^{2}$

Because a specific standard of communication has not been determined, developers can freely choose the protocol and speed of communication. They can also choose to use any legally allocated frequencies. Consequently, various modes of communication exist, which results in incompatibility between some satellites and ground stations. However, over $80 \%$ of the satellites have data rates lower than $10 \mathrm{kbps}$, which is relatively low compared with those of the wireless networks available on the ground.

\subsubsection{Duration of communication}

Communication is enabled only when the satellites enter the visibility region of their ground stations. The frequency of entering the region and the duration of being in the region depend on the orbit of each satellite and the location of the ground station. If only one ground station is available, communication is possible for only $30-100 \mathrm{~min} / \mathrm{d} .{ }^{(8)}$ 


\subsection{A new concept of Internet network in space}

The limitations of the traditional method of satellite communication have lead to the situation in which no network service is offered in space. This study proposes a new scheme using the Internet. The concept is that small satellites will be able to connect to the Internet while working in orbit, which will allow satellite owners control through the Internet. The satellite owners will not need to have their own stations. The rise of this standard may encourage the COTS system to be available in the market, which will help satellite developers decrease the risk of failure of their communication systems.

To expand the Internet into space, two main parts must be developed: a communication system for small satellites and an Internet gateway. All other satellite systems remain the same except the communication system that should follow the new concept. The Internet gateway needs to work both as a ground station and a gateway to the terrestrial Internet. The structure of the concept is shown in Fig. 3.

In this concept, satellites will have to formalize data into an application layer for the Internet protocol that is able to connect to the Internet, e.g., HTTP, FTP, or simple mail transfer protocol (SMTP), and to identify the IP address or domain name of receivers. They will then send this data to an Internet gateway on the ground. As the medium between a satellite and the ground is a radio link, we introduced LoRa, which stands for "Long Range", a technology that replicates in the first and second layers of open system interconnection (OSI) using LoRa media access control (LoRa MAC) and a low-power wide-area network (LPWAN) as standards. The details of LoRa are explained in the next section.

An Internet gateway acts as a network address translation (NAT) server. It forwards data from a satellite to the Internet and pushes data to reach their destinations. It also forwards commands from destinations to satellites in orbit.



Fig. 3. (Color online) New concept for the Internet in space. 
When a satellite changes position, it connects to other suitable gateways and works with the same concept to exchange data. With this concept, satellite owners do not need to build their own ground station but may still access the satellite most of the time over the Internet. The satellites themselves are similar to devices on the ground that perform activities through the Internet such as writing a post on facebook, submitting email, and sending data to store in databases.

\subsection{Internet network in space with LoRaWAN}

LoRaWAN is a type of LPWAN developed by the LoRa ${ }^{\mathrm{TM}}$ Alliance. It is a standard designed for a network of the Internet of Things (IoT) that has the ability to transmit data via radio frequencies with a maximum power of $30 \mathrm{dBm}$. It is able to communicate over a distance of up to $100 \mathrm{~km}$ while using low power, and one gateway can communicate to many devices in its coverage area. These features are appropriate for application to a prototype for the Internet of satellites. LoRaWAN has defined standards in its physical and MAC layers, and has features that are described in the following.

\subsubsection{RF in industrial scientific medical (ISM) band}

LoRaWAN uses ISM band frequency, which is an unlicensed band, in its communication. The users are allowed to employ the frequency and develop devices under the regulations of their country including the actual frequency band and maximum transmitting power. LoRaWan has been defined in regulations as shown in Table 1. To apply the ISM band in this research, as it is an unlicensed band, the satellite developers and gateway setters do not need to ask for permission. It provides a way to increase the number of gateways and expand coverage area.

\subsubsection{LoRaWAN RF modulation}

LoRa is a proprietary spread spectrum modulation scheme, which is a derivative of chirp spread spectrum (CSS) modulation and trades data rate for sensitivity within a fixed channel bandwidth. It uses a variable data rate utilizing orthogonal spreading factors (SFs), which allow the system designer to trade data rate for range or power to optimize network performance for a constant bandwidth. ${ }^{(10)}$

Table 1

LoRa frequency in ISM band. ${ }^{(9)}$

\begin{tabular}{lcc}
\hline Standard & Frequency & Max EIRP* \\
\hline EU 863-870 & $863-870 \mathrm{MHz}$ & $20 \mathrm{dBm}$ \\
US 902-928 & $902-928 \mathrm{MHz}$ & $30 \mathrm{dBm}$ \\
CN779-787 & $779-787 \mathrm{MHz}$ & $10 \mathrm{dBm}$ \\
EU433 & $433.05-434.79 \mathrm{MHz}$ & $10 \mathrm{dBm}$ \\
\hline
\end{tabular}

*EIRP: effective isotropic radiated power 


\subsubsection{LoRaWAN MAC}

LoRaWAN MAC can be mapped to the second and third layers of the OSI model. It is implemented on top of LoRa or frequency-shift keying (FSK) modulation in ISM radio bands. LoRaWAN protocols are defined by the LoRa Alliance and formalized in the LoRaWAN specifications.

\section{Prototype Implementation}

As proof of the concept of the Internet in space, we developed a CubeSat prototype, a small satellite using COTS devices similar to other CubeSat developments such as CUTE-1.7 + APD II, and a gateway. These prototypes are used to demonstrate the functions described in the next section.

\subsection{CubeSat prototype with LPWAN communication}

We redesigned the CubeSat previously developed by Geo-Informatics and Space Technology Development Agency (GISTDA) and Mahanakorn University of Technology (MUT) by modifying the software of the data handling and communication systems (Figs. 4 and 5). However, the main missions of the CubeSat are the imaging and compression of files into the JPEG format. These data are forwarded to a formalized FTP for data handling and then sent to a communication board that transmits data to a ground station in the form of LoRa MAC and Gaussian frequency-shift keying (GFSK) modulation.

\subsubsection{Communication subsystem}

The communication subsystem is an interface for the exchange of data between the CubeSat and a ground station. This subsystem has main and redundant systems for reliability. It uses an ATmega328 as a microcontroller to manage and communicate data in the form of LoRa MAC
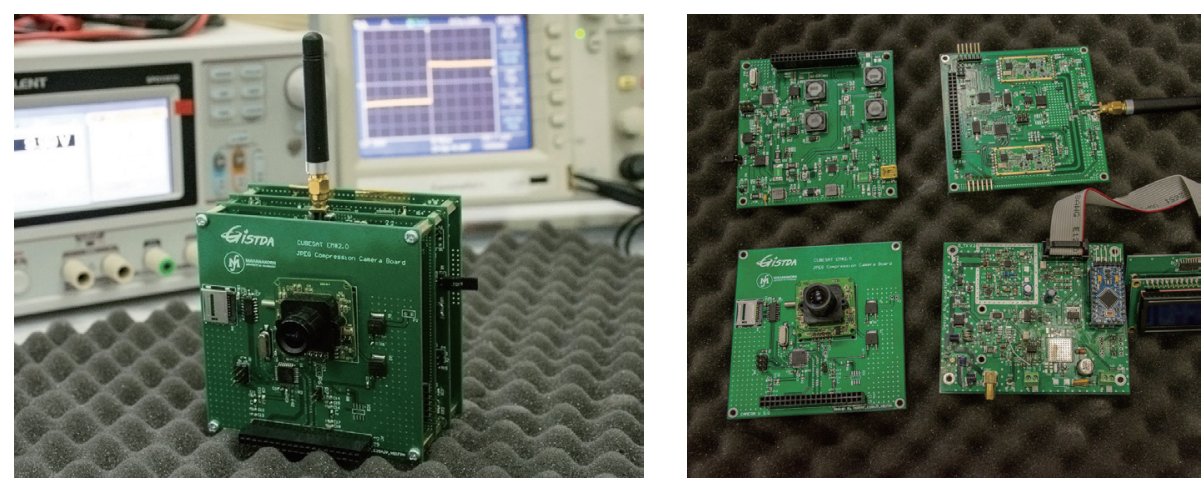

Fig. 4. (Color online) CubeSat prototype. 


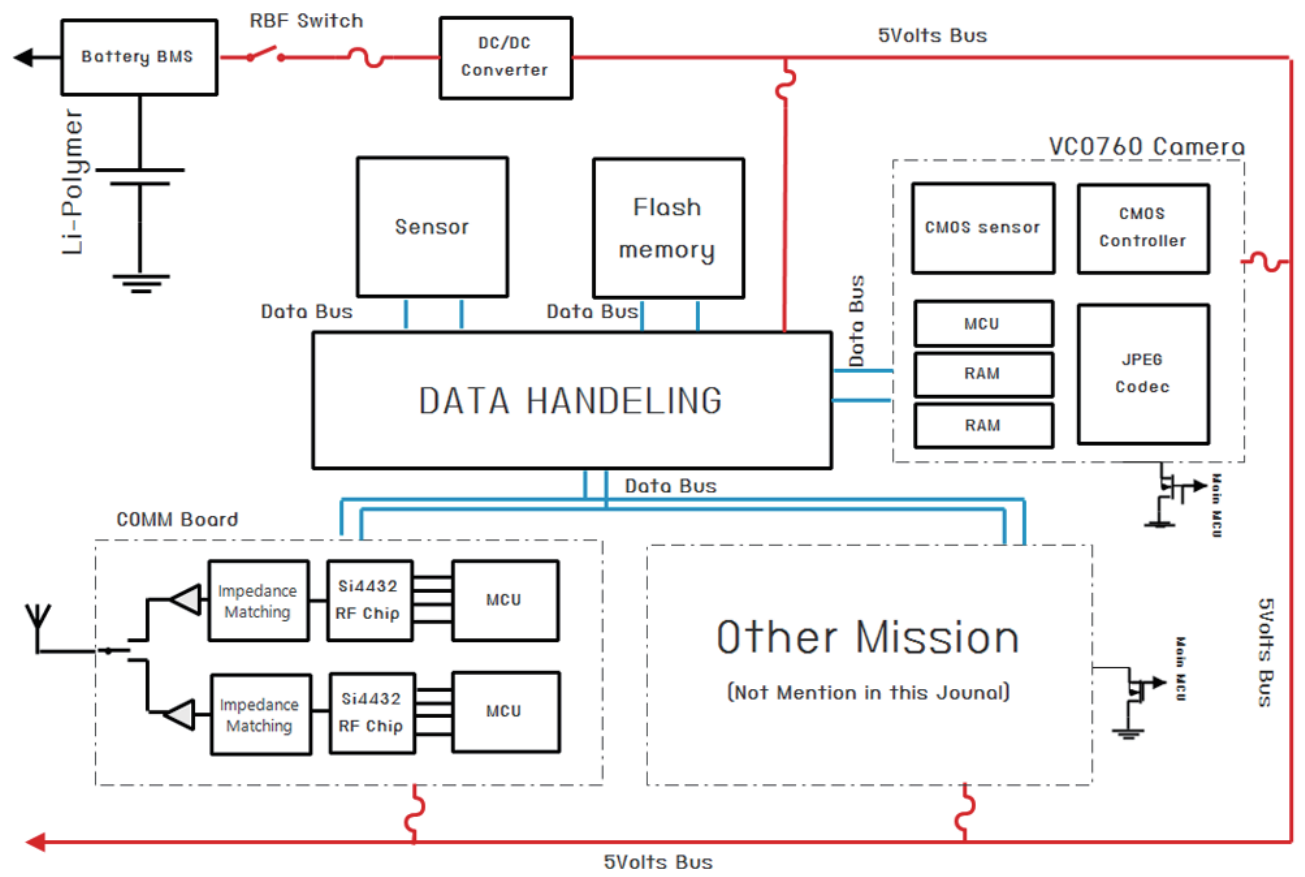

Fig. 5. (Color online) CubeSat diagram.

and a RFM23BP transceiver that modulates data into GFSK with a transmitting power of 30 dBm (Fig. 6).

\subsubsection{Mission subsystem}

The mission of the CubeSat is imaging using a VC0760 camera that contains a complementary metal-oxide-semiconductor (CMOS) sensor and a CMOS controller that convert light into digital signals. In addition, it involves a microcontroller, ram and JPEG codec process images, and compressed data sent to a universal asynchronous receiver/transmitter (UART) as a JPEG and to data handling via FTP (Fig. 7).

\subsection{LoRa communication prototype board}

The LoRa communication prototype board has been developed for testing LoRa communication and will be sent to near-space to test along with the CubeSat. The mission of this board is to cut the balloon rope by responding to telecommands from users (when they decide to end the mission) and sensing the temperature and global positioning system (GPS) position. We select a Ublox NEO-7 as the GPS module on this board. All data are processed at the ATMega328 microcontroller. For the communications section, we use a module LoRa610, which contains a Semtech SX1276 transceiver designed for LoRa communication. Its frequency was adjusted at $433.720 \mathrm{MHz}$ with a transmitting power of $100 \mathrm{~mW}$ and SF 12 with a bandwidth of $125 \mathrm{kHz}$, as shown in the diagram in Fig. 8. 

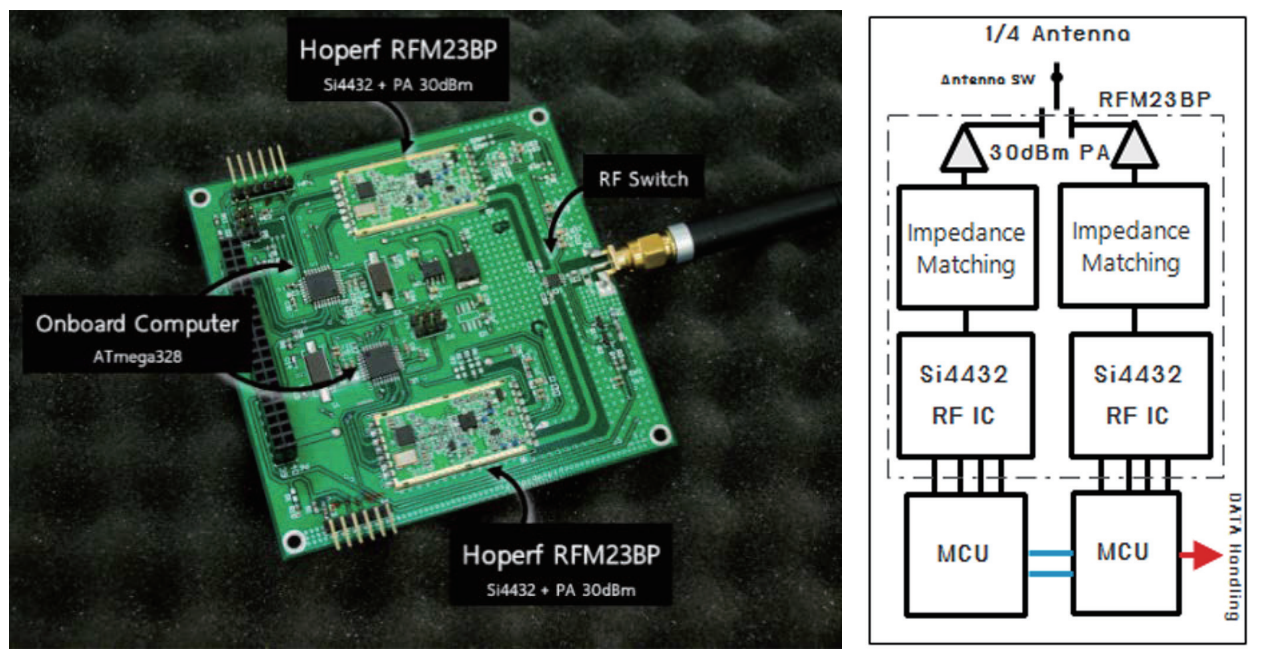

Fig. 6. (Color online) Communication subsystem board and diagram.
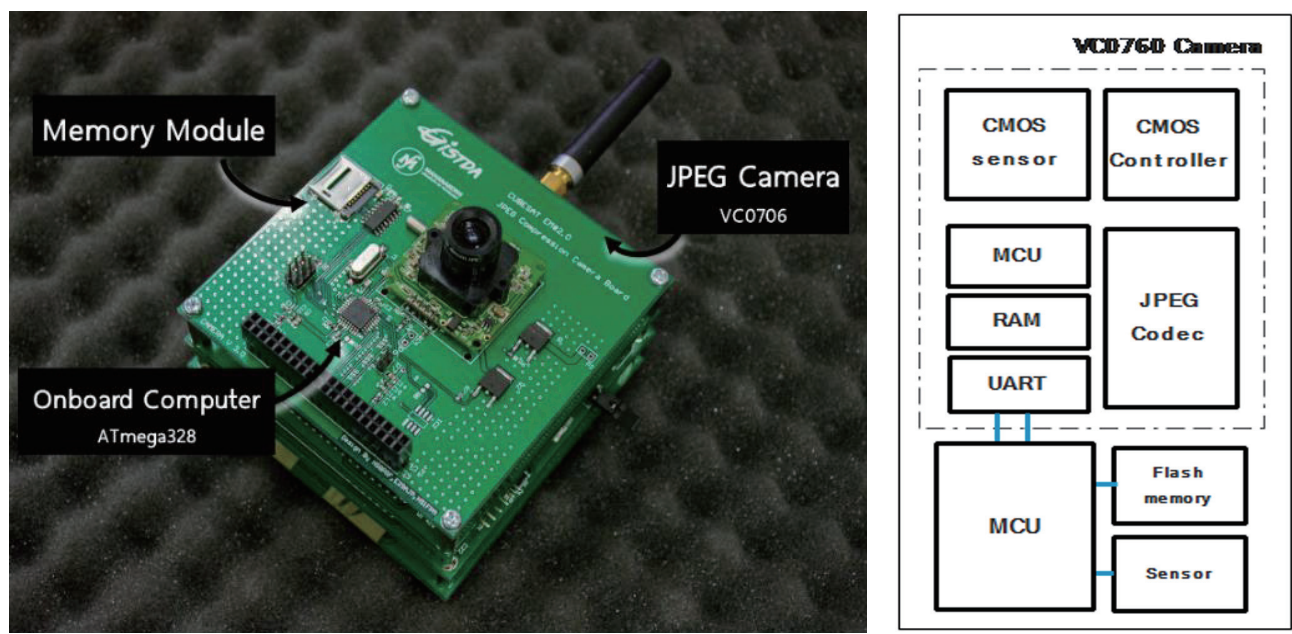

Fig. 7. (Color online) Mission subsystem board and diagram.
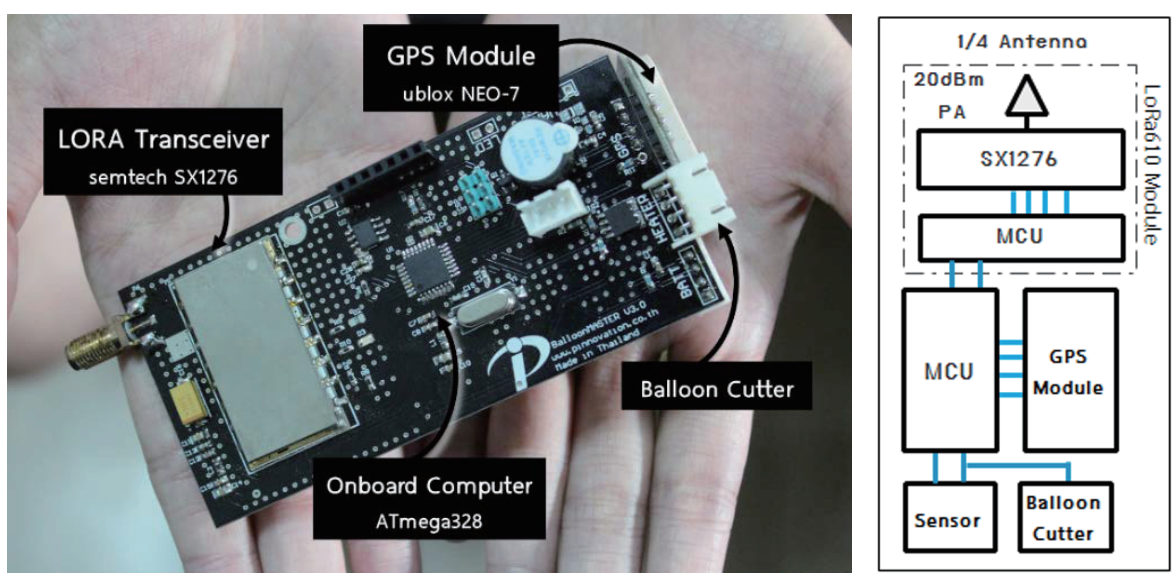

Fig. 8. (Color online) LoRa communication prototype board. 


\subsection{Internet gateway prototype}

The Internet gateway works as a ground station to communicate with the CubeSat and LoRa communication prototype board. It uses a Yagi-Uda antenna 7E to communicate at a frequency of $433.920 \mathrm{MHz}$, and it is able to communicate via LoRa modulation and GFSK as shown in Fig. 9.

The Internet gateway router unit is composed of two transceiver systems: 1) SI4432, which is the same module as that on the CubeSat, and 2) Semtech SX1276, which is the same module as that on the LoRa communication prototype board. Data from these two transceivers are demodulated and transformed from LoRa MAC to Ethernet with Ethernet module IEEE 802.3 as shown in the diagram in Fig. 10. This is a proper format for sending data to the terrestrial Internet. In addition, this system also has an antenna controller that allows remote control via the Internet.

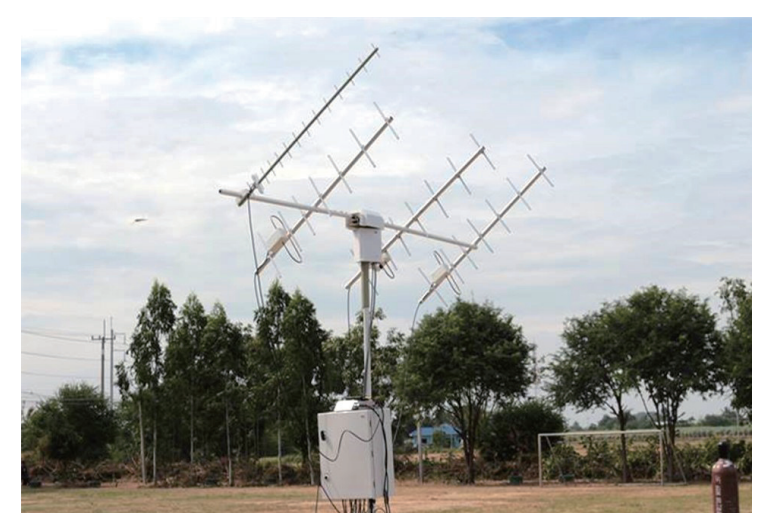

(a)

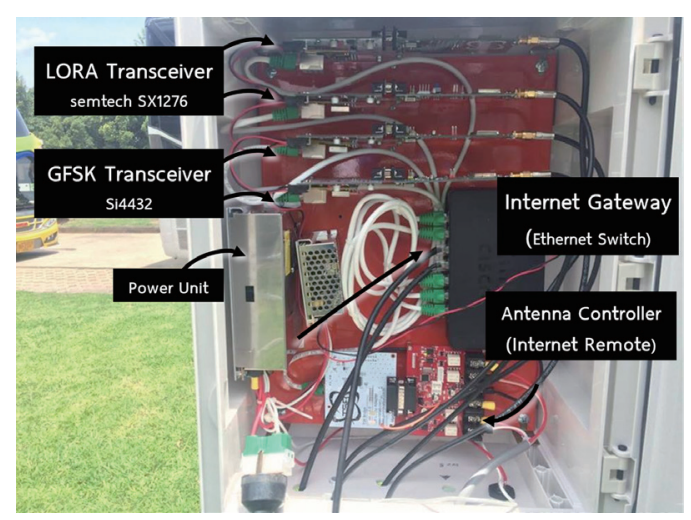

(b)

Fig. 9. (Color online) (a) Internet gateway prototype and (b) Internet gateway router unit.
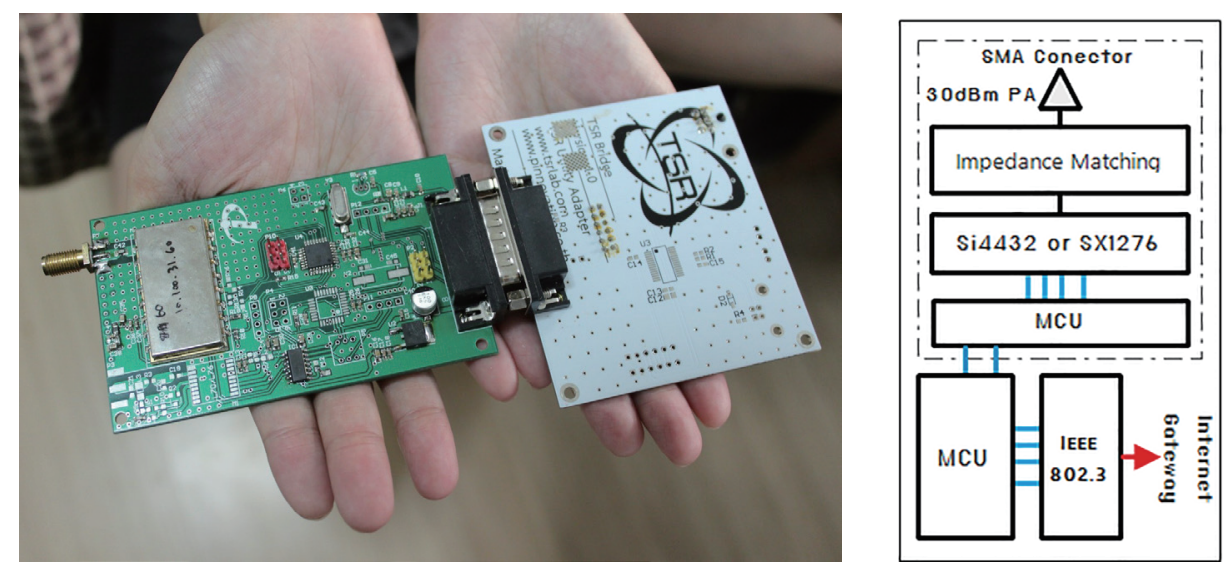

Fig. 10. (Color online) Internet gateway board. 


\subsection{Possibility of using LoRaWAN and GFSK for satellite communication by calculation}

In this section, we describe the calculation of distance between a satellite and a ground station that affects the link margin, which is the parameter that indicates the success of communication.

\subsubsection{Distance between satellite and gateway}

This research is of interest for small satellites that usually remain in LEO. The period that satellites travel over a ground station is called visibility. During visibility, the distance between a satellite and a ground station changes with the elevation of the satellite that can be calculated as

$$
D=R_{e}\left[\sqrt{\left(\frac{H+R_{e}}{R_{e}}\right)^{2}-\cos ^{2}(\varepsilon)}-\sin (\varepsilon)\right]
$$

where $D=$ distance between the satellite and the station at an elevation $\varepsilon(\mathrm{km}), R e=$ earth radius $(\mathrm{m})$ with a value of $6378 \mathrm{~km}, H=$ altitude of the satellite in orbit (m), and $\varepsilon=$ elevation (degrees).

$91 \%$ of all satellites in orbit usually remain at an altitude of $600 \mathrm{~km}$ or lower. ${ }^{(3)}$ Using Eq. (1) to calculate the elevation beginning at $30^{\circ}$, we found that the maximum distance between the satellite and the station is $1075 \mathrm{~km}$.

\subsubsection{Free-space path loss (FSPL)}

FSPL is the attenuation of radio energy between a transmitter and a receiver that results from many factors, e.g., the obstacle-free, line-of-sight path through free-space (usually air). We can calculate FSPL as

$$
F S P L=32.45+20 \log (D)+20 \log (f),
$$

where $D=\operatorname{distance}(\mathrm{km})$ and $f=$ frequency $(\mathrm{MHz})$.

\subsubsection{Link margin}

The link margin is the difference between a receiver's sensitivity and the expected minimum received power. It is used to determine the success of a communication and can be calculated using Eq. (3). In the case of applying LoRa SF 12, which achieves its highest sensitivity with a bandwidth of $125 \mathrm{kHz}$, we obtain a sensitivity of $-137 \mathrm{~dB}$ (according to the Semtech SX1276 datasheet). ${ }^{(11)}$ If we neglect transmission gain and receiver gain, a link margin with different standards is obtained as shown in Table 2.

Link Margin $=\left(-\right.$ sensitivity + Max EIRP $\left.+G_{t}+G_{r}\right)-F S P L$, 
Table 2

Link margin and FSPL.

\begin{tabular}{lccc}
\hline Standard & FSPL at 1100 km & Max EIRP & Link margin \\
\hline EU 863-870 & 151 & $20 \mathrm{dBm}$ & 4 \\
US 902-928 & 152 & $30 \mathrm{dBm}$ & 13 \\
CN 779-787 & 150 & $10 \mathrm{dBm}$ & -5 \\
EU 433 & 145 & $10 \mathrm{dBm}$ & 0 \\
\hline
\end{tabular}

where $G t=$ transmit antenna gain, $G r=$ receiver antenna gain, Max EIRP = maximum power of transmitter, and $F S P L=$ free-space path loss.

EU863-870 and US 902-928 have sufficient link margins for communication. We therefore assume that it is possible to use LoRa as a link between ground and a satellite.

\section{Demonstration of Internet Network in Space by Near-space Flight}

To demonstrate the concept of Internet in space described in Sect. 2, this project modified a CubeSat prototype as well as built a new Internet gateway prototype to illustrate this concept. The objective is to demonstrate the possibility of implementating a real system to work in a real situation.

The demonstration included the Internet gateway that works as a ground station and links Internet to space. The CubeSat was sent in near-space to an altitude of $30 \mathrm{~km}$ above sea level via a high-altitude balloon (HAB). The objectives were to test the data link from the CubeSat to the Internet on earth and evaluate the connection between the CubeSat and the Internet gateway.

\subsection{HAB for the experiments}

An HAB is a huge balloon made from Latex, usually filled with helium, a lighter-thanair gas, that can rise into the stratosphere at altitudes up to $30 \mathrm{~km}$, the so-called "near-space", without any propellant. It is an interesting platform to use for experiments because it can replicate the environment of space without actually going into space.

This experiment used a HAB with a diameter of $2.5 \mathrm{~m}$ as shown in Fig. 11; the HAB contained $7 \mathrm{~m}^{3}$ of helium that provided a gross lift of $5 \mathrm{~kg}$. After subtracting the payload (experiment) weight of $2 \mathrm{~kg}, 3 \mathrm{~kg}$ of free lift was retained. This platform can transport a payload to a high altitude at a speed of $4 \mathrm{~m} / \mathrm{s}$.

When it was released from the ground, the HAB brought the payload to a high altitude gradually. It traveled according to the wind direction and wind speed at that altitude, which cannot be controlled. The total flight path is shown in Fig. 12. It took about $2 \mathrm{~h}$ to ascend to an altitude of $30 \mathrm{~km}$, and the balloon expanded gradually as a function of air pressure, which decreases with increasing altitude.

Thus, when the balloon expanded, the thickness of the balloon skin decreased until it reached a critical value at which the pressure of the gas inside exceeded the strength of the elastic balloon material. Then, the balloon burst at an altitude of $30 \mathrm{~km}$ and the payload fell back to the ground by parachute, which spent about $2 \mathrm{~h}$. The distance between the sites of the 


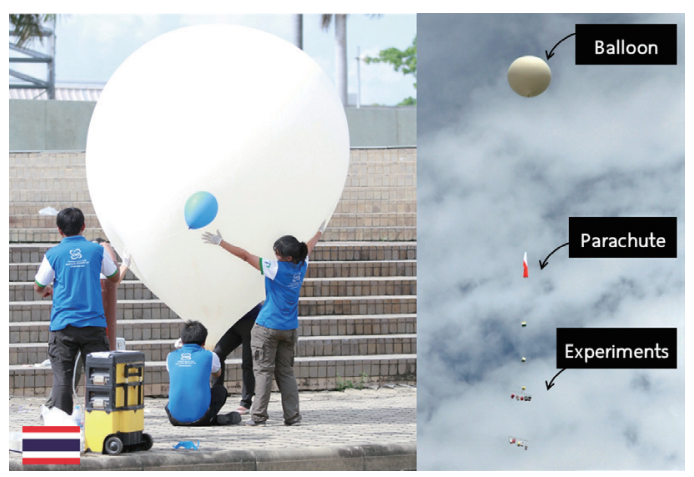

Fig. 11. (Color online) HAB with CubeSat prototype attached.

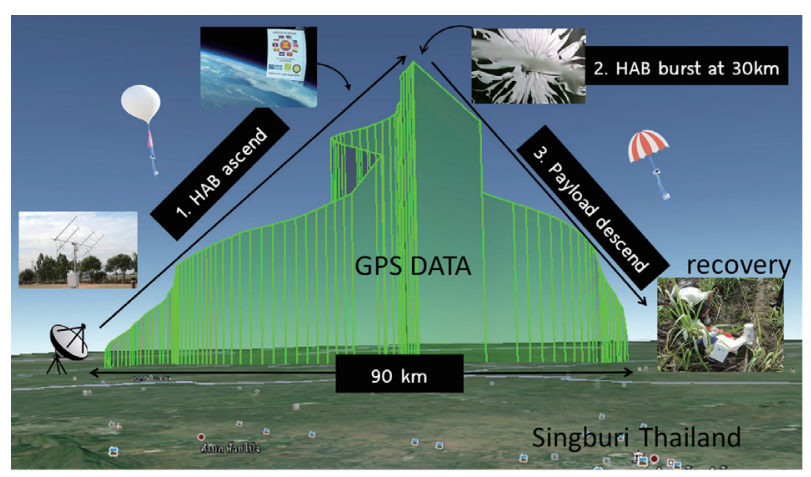

Fig. 12. (Color online) Flight path of HAB.

release and recovery of the parachute was about a hundred $\mathrm{km}$, which depended on the wind speed and direction on the day of the test.

\subsection{Diagram experimental concept}

The CubeSat and Internet gateway described in Sect. 3 were used in a real situation on 5 December 2016 (Fig. 13). The system was installed according to the diagram shown in Fig. 14.

The demonstration included the installation of the Internet gateway system at a ground station that connected the CubeSat and terrestrial Internet while the CubeSat was launched into near-space with a HAB. The objectives of this project were to test the connection between the CubeSat and the terrestrial Internet. The CubeSat was set to send telemetry and aerial photographs in real time through the Internet. Data should be broadcast to all online users, and the CubeSat should allow the controller to access it through the Internet as well.

The mission of this flight was to bring a message of condolence for His Majesty King Bhumibol Adulyadej to the highest altitude and then photograph this message with the view of near-space. Then, the data should be sent back using the Internet protocol that could be broadcast live on television (TV) and Facebook.

The CubeSat worked for $3 \mathrm{~h}$ throughout the mission and sent telemetry of position, altitude, temperature, and aerial photographs to the gateway. All data were uploaded to the Internet in real time. The CubeSat delivered two types of data: 1) CubeSat's status in Java script object notation (JSON) format and in HTTP protocol, sent via LoRa modulation SF 12 with a transmitting power of $15 \mathrm{dBm}$, and 2) aerial photographs by FTP protocol through modulation GFSK at a speed of $9600 \mathrm{bps}$ and a transmitting power of $30 \mathrm{dBm}$. Both data were sent at a frequency of $433.920 \mathrm{MHz}$, one at a time, according to the programing sequence.

The gateway also used a frequency of $433.920 \mathrm{MHz}$ to link to the CubeSat using a Yagi-Uda antenna with a transmitting power of $10 \mathrm{dBi}$. It demodulated data from LoRa and the GFSK format, then transformed LoRa MAC to Ethernet to deliver them to satellite controllers and the Thairat TV server. The Thairat TV server processed the images and added graphics into the information before uploading it online to Facebook and broadcasting it on TV. 


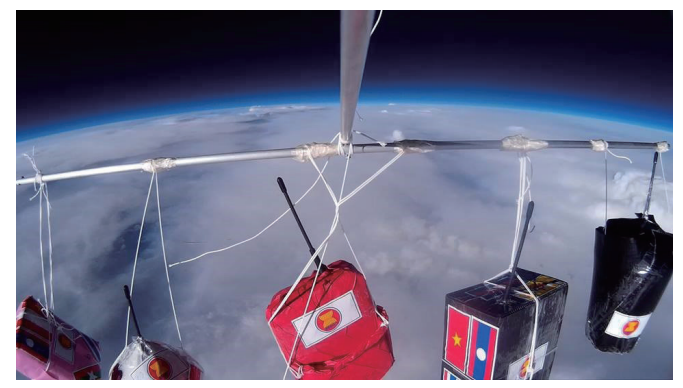

(a)

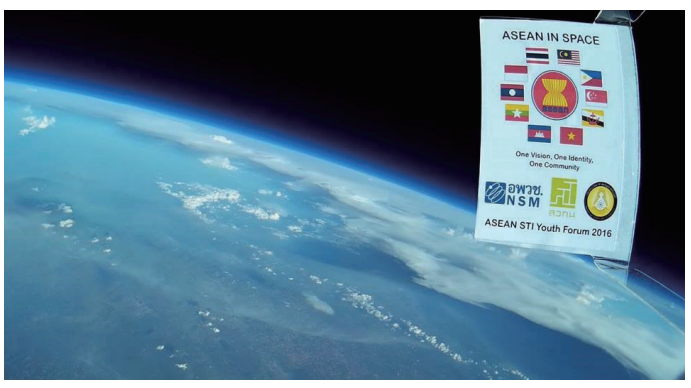

(b)

Fig. 13. (Color online) (a) Prototype of CubeSat working in near-space and (b) view at the highest altitude.

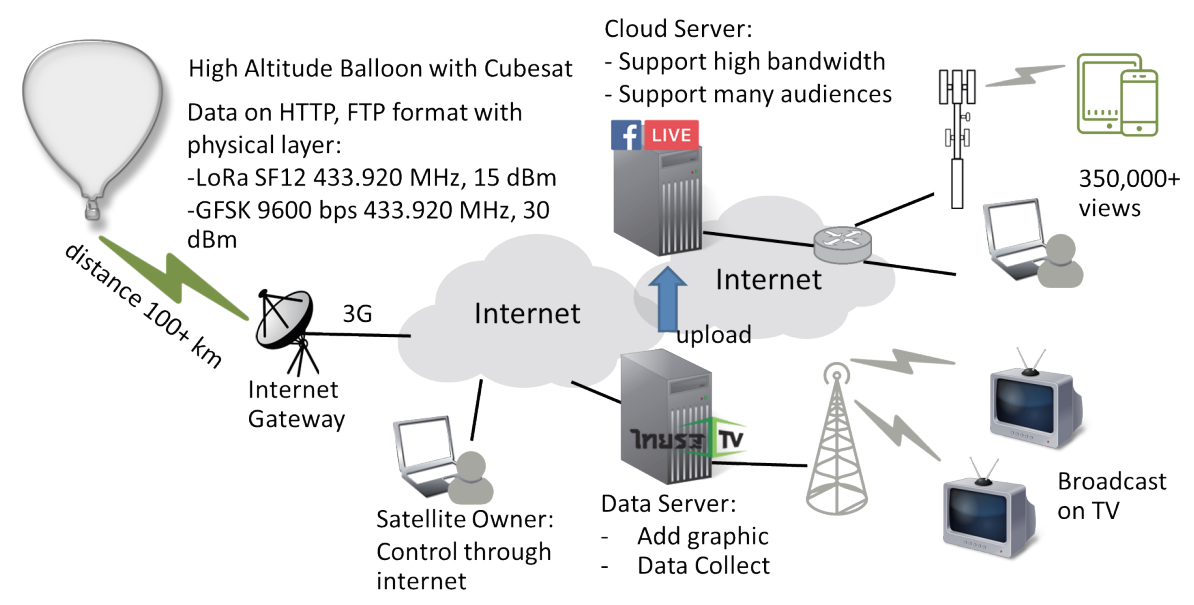

Fig. 14. (Color online) Diagram of concept demonstration.

During the mission, the satellite owners were able to control the CubeSat using the Internet. They could receive CubeSat's status and images as well as change some configurations on the CubeSat by sending control commands back through the Internet without being at a ground station.

\subsection{Results}

\subsubsection{Experimental results}

We succeeded in building a connection between the CubeSat and a gateway, which were able to communicate seamlessly. Data from the CubeSat can be delivered through the Internet in real time.

The images of the message of condolence taken in near-space were broadcast live online both on Facebook and on TV. Even though there were other TV programs running, CubeSat's images were presented on the right-hand side of the screen and CubeSat's altitude and temperature on the left-hand side allowed users to follow the balloon in real time as shown in Fig. 15. 

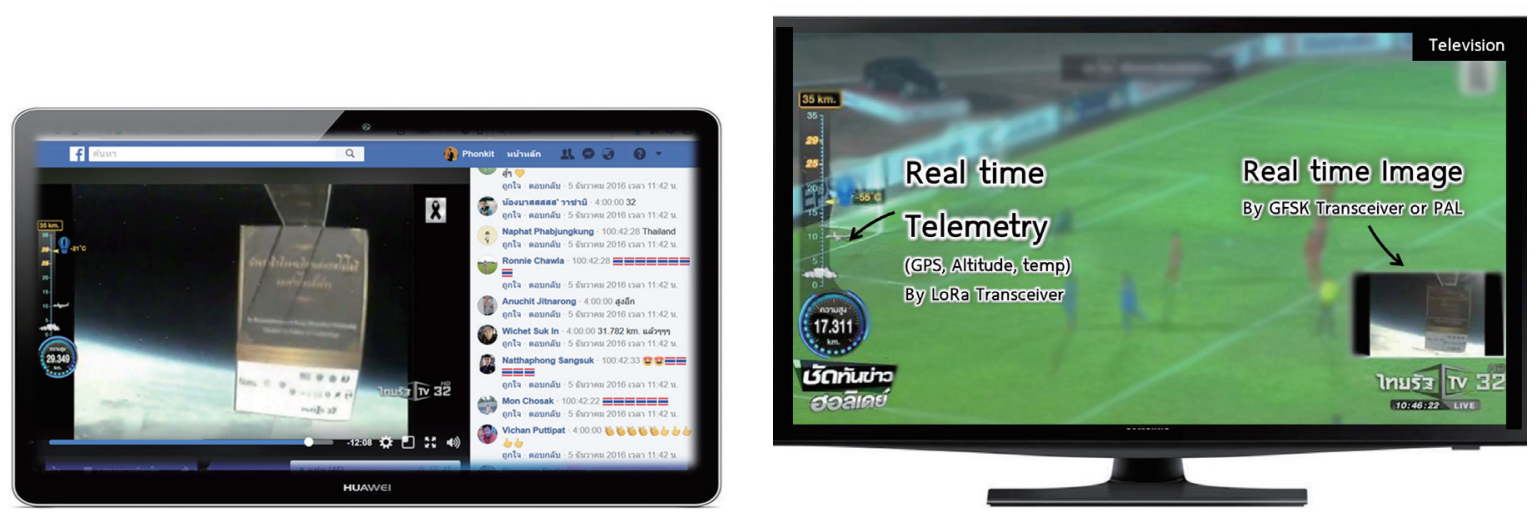

Fig. 15. (Color online) Real-time telemetry from CubeSat broadcast live on Facebook and TV.

They were more than 350000 views on Facebook Live, and they were also live broadcasts on $\mathrm{TV}$ at the same time for $3 \mathrm{~h}$ throughout the mission. We were able to send control commands to change some configurations on the CubeSat.

However, this demonstration is just a beginning in the development of the concept of using the Internet in space. To implement this concept for working in actual space, one must be concerned about the link budget and link margin that must be sufficient for communicating from space to ground as described in Sect. 3.4.

\subsubsection{Sensor data from CubeSat}

This flight used sensors to collect data from near-space, which included temperatures inside, outside, and at the battery of CubeSat, carbon dioxide $\left(\mathrm{CO}_{2}\right)$ concentration, air pressure, and solar density. The data are described as follows.

\section{Temperature data}

This flight attached 3 temperature sensors in different places, namely, outsidet, inside, and at the battery of the CubeSat. We observed the temperature at the battery because the battery is sensitive to the temperature. When the HAB was released from the ground and reached a higher altitude, the temperature decreased, particularly the temperature outside, while the temperature inside became more stable as shown in Fig. 16. At the battery, we attached a heater to maintain the temperature to be not less than 0 .

However, the temperature outside the CubeSat depended on the sensor direction into or out of the sunlight. The lowest temperature in this flight was $-37^{\circ} \mathrm{C}$. This data will be beneficial for the future CubeSat design and development to operate potentially long term in space.

\section{Concentration of carbon dioxide}

The nondispensive infrared (NDIR) $\mathrm{CO}_{2}$ sensor was installed outside the CubeSat to measure the $\mathrm{CO}_{2}$ concentration in atmosphere at different altitudes. The sensor can measure the $\mathrm{CO}_{2}$ 


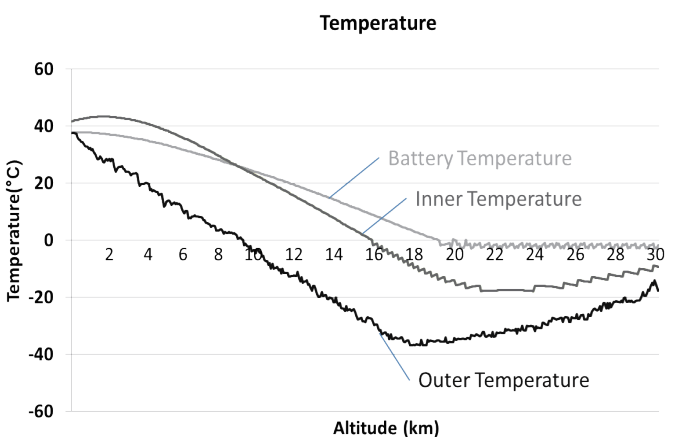

Fig. 16. (Color online) Temperatures obtained at different altitudes.

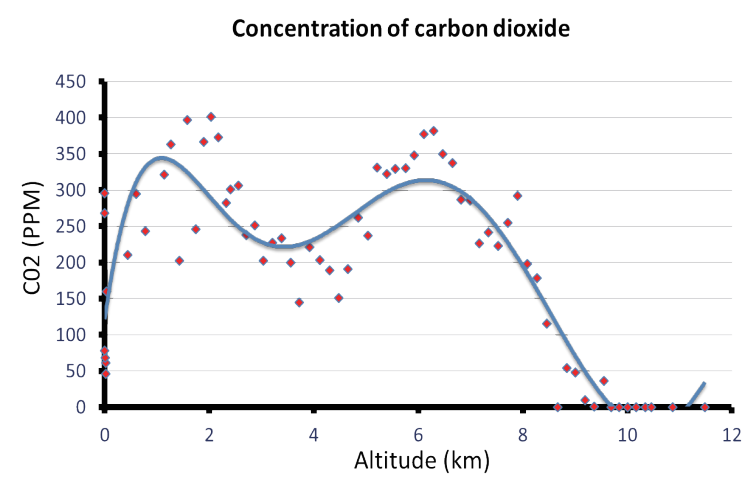

Fig. 17. (Color online) $\mathrm{CO}_{2}$ concentrations obtained at different altitudes.

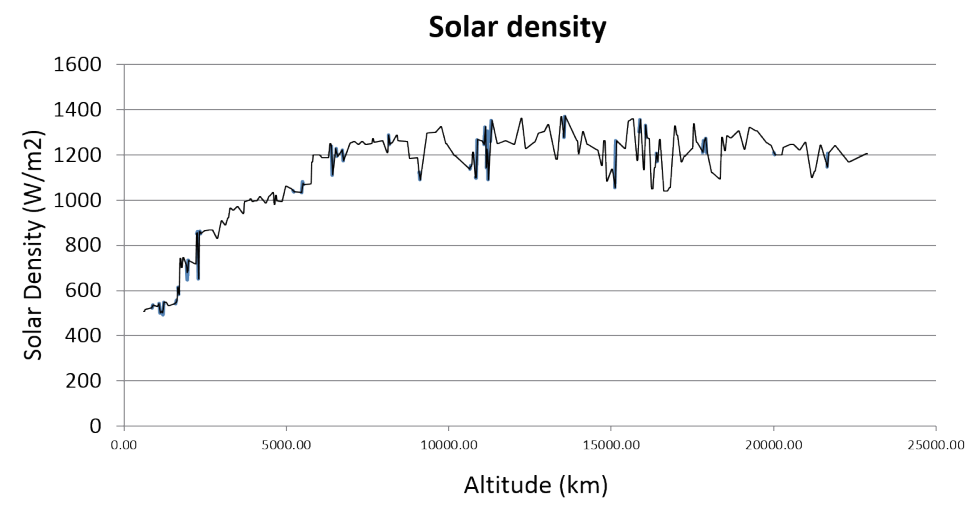

Fig. 18. (Color online) Solar densities obtained at different altitudes.

concentration in the range from 0 to 5000 parts per million (ppm). The graph in Fig. 17 shows high $\mathrm{CO}_{2}$ concentrations at altitudes of 2 and $6 \mathrm{~km}$. It might be possible that the greenhouse gas usually concentrated at these altitudes. However, the sensor stopped working after the altitude of $12 \mathrm{~km}$ due to some technical problems.

\section{$\underline{\text { Solar density }}$}

To measure solar density at near-space, we installed the photovoltaic that connects with the load and then measured the power supplied to the load by considering the area of the photovoltaic and its efficiency. The solar density unit is $\mathrm{W} / \mathrm{m}^{2}$.

The graph in Fig. 18 shows solar densities obtained at different altitudes. We found that the solar density increased at higher altitudes and became stable at an altitude of about $10 \mathrm{~km}$. The maximum value corresponds to a solar constant of $1353 \mathrm{~W} / \mathrm{m}^{2}$, which has been recorded by National Aeronautics and Space Administration (NASA) and accepted as a standard value. ${ }^{(12)}$ 


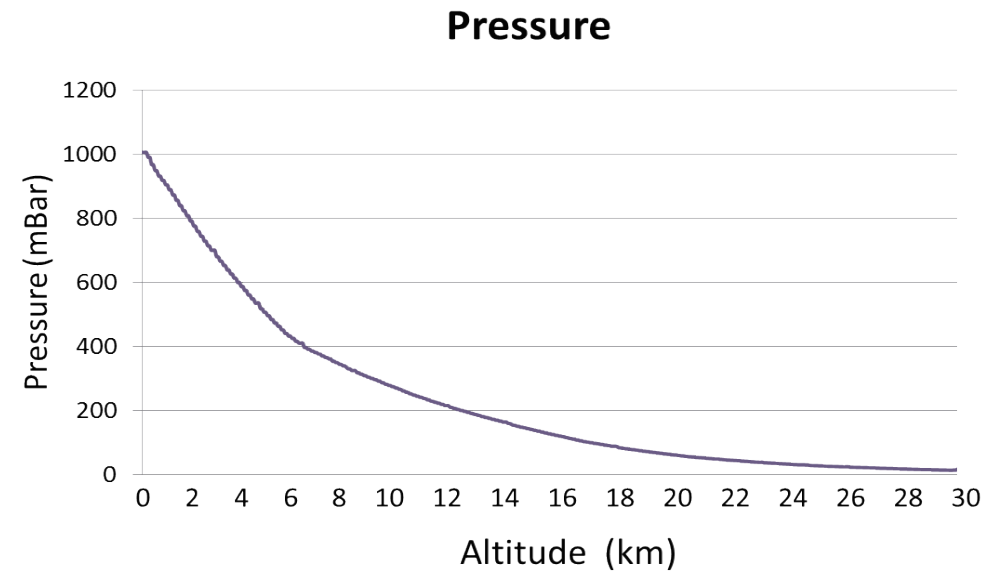

Fig. 19. Air pressures obtained at different altitudes.

\section{Air pressure}

This flight installed the air pressure sensor outside the CubeSat to measure air pressure at different altitudes. The graph in Fig. 19 shows that air pressure decreased with increasing altitude and its value was close to 0 at the highest altitude of $30 \mathrm{~km}$.

\section{Conclusions}

In this paper, we propose a new concept for small satellite communication by implementing an Internet in space to solve the problems of traditional communication that does not have any service network in space. Developers need to implement their own communication, which has problems of low coverage and does not function very well.

The concept of Internet in space addresses all these issues by a new method of communication that can be applied globally. Satellite developers do not need to have their own ground stations but still could access their satellites most of the time through the Internet. For a radio communication link, this research introduced LoRa technology, because LoRa uses the ISM band that is convenient for both satellite developers and gateway setters to implement without a frequency license. In addition, the link margin is also sufficient for communicating from ground to space using standard EU 863-870 and US 902-928. ${ }^{(9)}$

This work involved building a CubeSat, a small satellite, and an Internet gateway to demonstrate the entire system. The CubeSat was launched with a HAB into near-space and a gateway was installed to connect to the terrestrial Internet. After testing for $3 \mathrm{~h}$, the result showed that the CubeSat could communicate using an Internet protocol and submit data from near-space to the terrestrial Internet by broadcasting real-time aerial photographs on TV and Facebook Live that had more than 350000 viewers throughout the mission. The records of all activities are on the Thairath youtube channel. ${ }^{(13)}$

The data collected from sensors, which include temperature, solar density, $\mathrm{CO}_{2}$ concentration, and air pressure, are useful for us to study the environmental conditions at 
altitudes of $0-30 \mathrm{~km}$. In addition, the data at the highest altitude is similar to the condition of the outer space. This will be helpful for our team to understand the space environment and will help us design and develop a CubeSat that can stay in orbit for a long time.

For future development, our research team will test this concept with an actual satellite in space. We are developing a CubeSat called "Spacebox-STEP1" that will be launched into actual space by a rocket. We are also modifying an Internet gateway to be able to communicate to a CubeSat in orbit. The Spacebox project will be a prototype for the future development of the Internet in space.

\section{Acknowledgments}

This work was supported by the FY2018 Thesis Grant from the National Research Council of Thailand (NRCT). We would like to thank especially the Thailand Space and Aeronautic Research (TSR) team for their participation in the development of Cubesat "Spacebox-STEP1".

\section{References}

1 N. Miyashita, M. Iai, K. Omagari, K. Imai, H. Yabe, K. Miyamoto, T. Iljic, T. Usuda, K. Fujiwara, S. Masumoto, Y. Konda, S. Sugita, T. Yamanaka, K. Konoue, and S. Matunaga: Proc. Int. Astronautical Congr. http://1ss.mes. titech.ac.jp/ssp/cute1.7/paper/iac2005.pdf

2 S.Nakasuka, N. Sako, H. Sahara, Y. Nakamura, T. Eishima, and M. Komatsu: Acta Astronaut. 66 (2010) 1099. https://www.sciencedirect.com/science/article/pii/S0094576509004810

3 M. Swartwout: Swartwout's CubeSat database: https://sites.google.com/a/slu.edu/swartwout (accessed November 2017).

4 P. Halis, J. Virgili-Llop, and M. Romano: JoSS 5 (2016) 513. https://goo.gl/ZM8fCN

5 Z. Qu, G. Zhang, H. Cao, and J. Xie: IEEE Access 5 (2017) 18391. https://ieeexplore.ieee.org/ document $/ 8002583$

6 T. R. Henderson and R. H. Katz: IEEE JSAC 17 (1999) 326. https://goo.gl/sL4Xvo

7 B. Klofas: CubeSat Communications System Table: http://www.klofas.com/comm-table (accessed November 2017).

8 M. Krynitz and K. Olafsson: 34th Asian Conf. Remote Sensing, Bali, Indonesia (2013) 291-292. https://goo.gl/ SlugL5

9 LoRa Alliance Technical Committee Regional Parameters Workgroup, LoRaWAN ${ }^{\mathrm{TM}} 1.1$ Regional Parameters (January 2018). https://goo.gl/tkPKbK

10 N. Sornin, M. Luis, T. Eirich, T. Kramp, and O. Hersent: LoRaWANTM Specification (V1.1, October 2017). http://net868.ru/assets/pdf/LoRaWAN-v1.1.pdf

11 SEMTECH SX1276 datasheet: http://www.semtech.com/wireless-rf/rf-transceivers/sx1276 (accessed November 2017) p. 21.

12 K. N. Liou: An Introduction to Atmospheric Radiation (Academic press, Cambridge, 2002) p. 38.

13 The record of experiment of balloon flight broadcast on facebook live on 5 December 2016 by Thairath TV: https:/www.youtube.com/watch?v=iRNsJ8mv7zM\&t=1772s 